\title{
Charge-exchange isobaric resonances
}

\author{
Yu. S. Lutostansky,* \\ ${ }^{1}$ National Research Centre "Kurchatov Institute", 123182, Moscow, Russia
}

\begin{abstract}
Three types of the charge-exchange isobaric resonances - giant Gamow-Teller (GTR), the ana$\log (\mathrm{AR})$ and pygmy (PR) ones are investigated using the microscopic theory of finite Fermi systems and its approximated version. The calculated energies of GTR, AR and three PR's are in good agreement with the experimental data. Calculated differences $\Delta E_{\mathrm{G}-\mathrm{A}}=E_{\mathrm{GTR}}-E_{\mathrm{AR}}$ go to zero in heavier nuclei indicating the restoration of Wigner SU(4)-symmetry. The average deviation for $\Delta E_{\mathrm{G}-\mathrm{A}}$ is $0.30 \mathrm{MeV}$ for the 33 considered nuclei where experimental data are available. The comparison of calculations with experimental data on the energies of charge-exchange pygmy resonances gives the standard deviation $\delta E<0.40 \mathrm{MeV}$. Strength functions for the ${ }^{118} \mathrm{Sn},{ }^{71} \mathrm{Ga},{ }^{98} \mathrm{Mo}$ and ${ }^{127} \mathrm{I}$ isotopes are calculated and the calculated resonance energies and amplitudes of the resonance peaks are close to the experimental values. Strong influence of the charge-exchange resonances on neutrino capturing cross sections is demonstrated.
\end{abstract}

\section{Introduction}

Charge-exchange isobaric states are manifested in the corresponding charge-exchange reactions such as $(v, e)$, $(p, n),(n, p),\left({ }^{3} \mathrm{He}, t\right),\left(t,{ }^{3} \mathrm{He}\right),\left({ }^{6} \mathrm{Li},{ }^{6} \mathrm{He}\right)$ and others, or in $\beta$-transitions in nuclei. Among these states, collective resonance excitations are of the most interest. The theoretical studies of these collective states have began with the first calculations of the giant Gamow-Teller resonance (GTR) [1] and other collective states [2] long before their experimental studies in charge-exchange reactions $[3,4]$. These collective states lying below the giant GTR [5] were called "pygmy" resonances (PR).

The most complete experimental information on the charge-exchange excitations in 9 tin isotopes with $A=112$ 124 were obtained in [6], where the $\operatorname{Sn}\left({ }^{3} \mathrm{He}, t\right) \mathrm{Sb}$ chargeexchange reaction at the energy $E\left({ }^{3} \mathrm{He}\right)=200 \mathrm{MeV}$ was used. The excitation energies $\left(E_{x}\right)$, widths $(\Gamma)$, and cross sections $\mathrm{d} \sigma / \mathrm{d} \Omega(\mathrm{mb} / \mathrm{sr})$ were measured for the analog, Gamow-Teller, and three pygmy resonances. Also some charge-exchange strength-functions $S(E)$ were measured earlier in $(p, n)$ and $\left({ }^{3} \mathrm{He}, t\right)$ reactions. The influence of the charge-exchange resonances on neutrino capturing cross sections was investigated also.

\section{Method of calculation}

Charge-exchange excitations of nuclei are described in the microscopic theory of finite Fermi systems (TFFS) by the system of equations for the effective field [7]

$$
\begin{aligned}
& V_{p n}=e_{q} V_{p n}^{\omega}+\sum_{p^{\prime} n^{\prime}} F_{n p, n^{\prime} p^{\prime}}^{\omega} \rho_{p^{\prime} n^{\prime}} \\
& V_{p n}^{h}=\sum_{p^{\prime} n^{\prime}} F_{n p, n^{\prime} p^{\prime}}^{\omega} \rho_{p^{\prime} n^{\prime}}^{h}
\end{aligned}
$$

\footnotetext{
*e-mail: lutostansky@yandex.ru
}

where $V_{p n}$ and $V_{p n}^{h}$ are the effective fields of quasi-particles and holes in a nucleus whereas $V_{p n}^{\omega}$ is the external chargeexchange field. The system of secular equations (1) is solved for allowed transitions with local nucleon-nucleon interaction in the Landau-Migdal form [7]

$$
F^{\omega}=C_{0}\left(f_{0}^{\prime}+g_{0}^{\prime}\left(\vec{\sigma}_{1} \vec{\sigma}_{2}\right)\right)\left(\vec{\tau}_{1} \vec{\tau}_{2}\right) \delta\left(\vec{r}_{1}-\vec{r}_{2}\right)
$$

with the parameters $f_{0}^{\prime}=1.35$ and $g_{0}^{\prime}=1.22$ as in [8].

The energies of charge-exchange excitations were calculated both in the self-consistent TFFS and in its approximate version [5], which allowed to obtain the analytical solutions for the most collective states. For the energies $E_{\mathrm{GTR}}$ and $E_{\mathrm{PR}}$, the solutions $\omega_{k}(k=0$ for GTR and $k=1,2,3$ for PR1, PR2, and PR3, correspondingly) divided by the average value $E_{\mathrm{ls}}$ of the spin-orbit splitting [8] at $\Delta E>E_{\mathrm{ls}}$, has the form

$$
\begin{aligned}
y_{k} & =\omega_{k} / E_{\mathrm{ls}}=\left(a_{k}+b_{k}\right) g_{k}^{\prime} x+ \\
& +\frac{b_{k}\left(1+b_{k} g_{k}^{\prime}\right) g_{k}^{\prime} x}{\left(a_{k}+b_{k}\right)\left(g_{k}^{\prime} x\right)^{2}+\left[1+2\left(a_{k}+b_{k}\right) g_{k}^{\prime}\right] / 3 A^{1 / 3}},
\end{aligned}
$$

where

$$
x=\Delta E / E_{\mathrm{ls}}, \Delta E=\frac{4}{3} \varepsilon_{\mathrm{F}} \frac{N-Z}{A} \mathrm{MeV}, \varepsilon_{\mathrm{F}} \approx 40 \mathrm{MeV} .
$$

Parameters $a_{k}, b_{k}$ and $g_{k}$ in (3) are defined as follows:

$$
\begin{gathered}
a_{k} \approx a \cdot p_{k}, \quad b_{k} \approx b \cdot p_{k}, \quad p_{k} \approx(k+1)^{-1}, \\
b=\frac{2}{3}\left[1-(2 A)^{-1 / 3}\right], \quad a+b=1
\end{gathered}
$$

and for $k>0$

$$
g_{k}^{\prime}=\frac{g_{0}^{\prime}}{1+\alpha_{k} \beta_{k} / 2}, \quad \alpha_{k}=\frac{p_{k} \beta_{k}}{1+2 g_{0}^{\prime} \beta_{k}}, \quad \beta_{k}=\sum_{m=1}^{k} p_{m} .
$$


Thus, all resonances, i.e. GTR $(k=0)$ and PR $k(k=1,2,3)$, are described by the single formula (3).

The energies of analog resonances (AR) were calculated within TFFS and also from the simple formulas for the difference $\Delta E_{\mathrm{C}}$ between the Coulomb energies of neighboring isobar nuclei

$$
\begin{gathered}
E_{\mathrm{AR}}=E_{x}\left(A R_{Z+1}\right)=\Delta E_{\mathrm{C}}-\delta Q, \\
\Delta E_{\mathrm{C}}=E_{\mathrm{C}}(A, Z+1)-E_{\mathrm{C}}(A, Z)=a_{\mathrm{C}} \frac{Z}{A^{1 / 3}}+b_{\mathrm{C}}
\end{gathered}
$$

where $\delta Q=Q_{\beta}+\Delta_{n p}$, and $\Delta_{n p}=0.78234 \mathrm{MeV}$ is the dif-

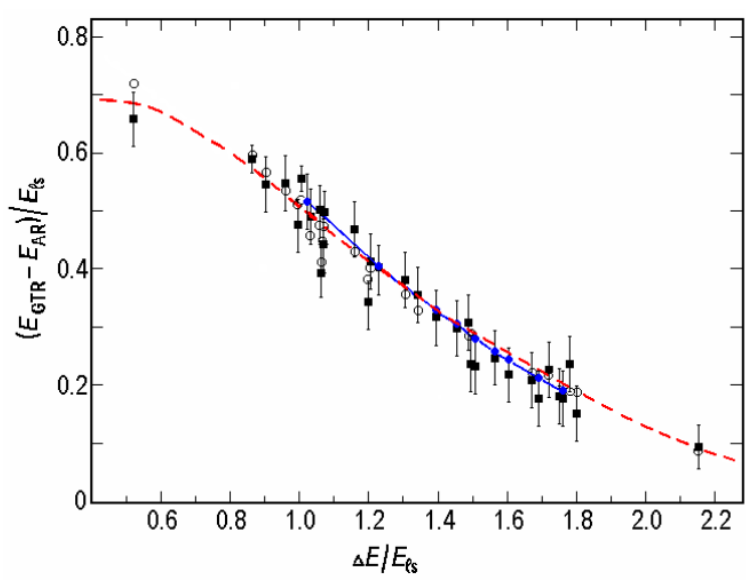

Figure 1. (Color online) The ratio $\Delta y_{0}(x)=\left(E_{\mathrm{GTR}}-E_{\mathrm{AR}}\right) / E_{\mathrm{ls}}$ as a function of parameter $x=\Delta E / E_{\mathrm{ls}}$. Open circles - results of calculations by Eq. (1). Black squares - experimental data. Blue dots connected by the blue line are the values for $\mathrm{Sn}$ isotopes. The dashed red line shows the function $\Delta y_{0}(x)$ calculated by Eq. (6) for nuclei located on the beta-stability line.

ference between the neutron mass and the mass of hydrogen atom. For all nuclei with $5 \leq A \leq 244$ we have obtained $a_{\mathrm{C}}=1416 \mathrm{keV}$ and $b_{\mathrm{C}}=-698 \mathrm{keV}$ [9] with the standard deviation $\delta E<100 \mathrm{keV}$. Deformation was taken into account as in [9], by introducing the correction to $\Delta E_{\mathrm{C}}=\Delta E_{\mathrm{C}}^{\mathrm{sph}}-\delta E_{\mathrm{C}}^{\mathrm{def}}$ with the deformation parameters $\beta_{2}$ and $\beta_{4}$ from [10].

\section{Results}

Differences of energies between Gamow-Teller and analog resonances $\Delta E_{\mathrm{G}-\mathrm{A}}=E_{\mathrm{GTR}}-E_{\mathrm{AR}}$ were calculated in TFFS for 33 target nuclei ${ }^{48} \mathrm{Ca},{ }^{60,64} \mathrm{Ni},{ }^{71} \mathrm{Ga}$, ${ }^{76} \mathrm{Ge},{ }^{82} \mathrm{Se},{ }^{90,91,92,94} \mathrm{Zr},{ }^{93} \mathrm{Nb},{ }^{44,96,97,98,100} \mathrm{Mo},{ }^{115} \mathrm{In}$, $112,114,116,117,118,119,120,122,124 \mathrm{Sn}, \quad{ }^{128,130} \mathrm{Te},{ }^{127} \mathrm{I},{ }^{136} \mathrm{Xe}$, ${ }^{150} \mathrm{Nd},{ }^{169} \mathrm{Tm}$, and ${ }^{208} \mathrm{~Pb}$ for which experimental data are available $[6,11]$. The results of theoretical calculations and experimental data are presented in Fig. 1. Strictly speaking, in Fig. 1 we display the calculated and extracted from experimental data the dimensionless ratio $y(x)=\Delta E_{\mathrm{G}-\mathrm{A}} / E_{\mathrm{ls}}$ as a function of parameter $x=\Delta E / E_{\mathrm{ls}}$. Moreover, the values of $\Delta y_{0}(x)$ calculated by the following equation:

$$
\begin{aligned}
\Delta y_{0}(x) & =\left(E_{\mathrm{GTR}}-E_{\mathrm{AR}}\right) / E_{\mathrm{ls}} \approx\left(g_{0}^{\prime}-f_{0}^{\prime}\right) x+ \\
& +b \frac{1+b g_{0}^{\prime}}{g_{0}^{\prime} x\left(1+c_{A} x^{-2}\right)}, \quad c_{A}=0.8 A^{-1 / 3}
\end{aligned}
$$

is also depicted in Fig. 1. Equation (6) is related with Eq. (3) where $k=0$ for the GTR energy. The standard r.m.s. deviation of the values calculated within TFFS Eq. (2) from the experimental data for the listed above nuclei is $\delta(\Delta \varepsilon) \leq 0.30 \mathrm{MeV}$ which is comparable to the accuracy of the $E_{\mathrm{GTR}}$ experimental data [6].

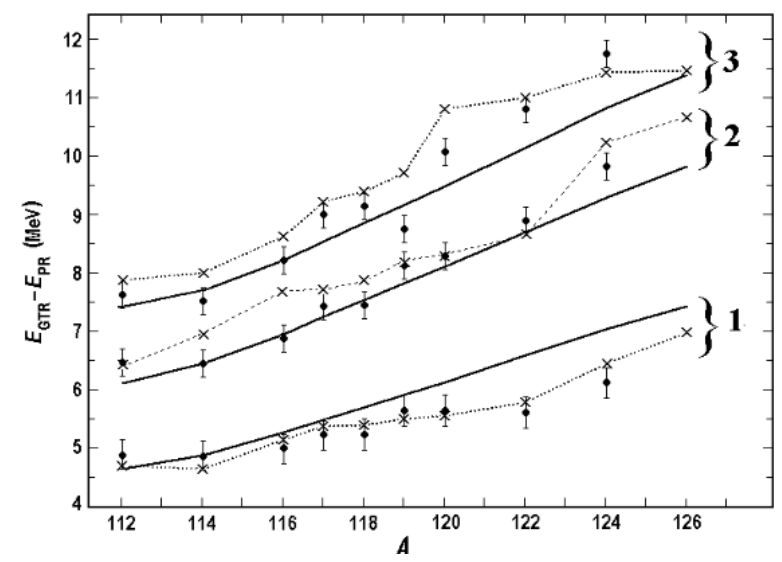

Figure 2. Differences $E_{\mathrm{GTR}}-E_{\mathrm{PR}}$ between the GTR energies and the energies of pygmy resonances in $\mathrm{Sn}$ isotopes as functions of the mass number $A$. Experimental data [6] are indicated by $\bullet$ with error bars; calculations by TFFS $-\times$ connected by dashed lines; calculations by Eq. (3) - solid lines. The digits 1, 2, and 3 indicate results and data for pygmy resonances PR1, PR2, and PR3, correspondingly.

Calculated differences $\Delta E_{\mathrm{G}-\mathrm{A}}$ go to zero in heavier nuclei (for ${ }^{208} \mathrm{~Pb} x=2.15$ ) indicating the restoration of Wigner SU(4)-symmetry [8]. Thus the analog and Gamow-Teller resonances belong to the same supermultiplet. AR energies were analyzed using both TFFS and simple Eqs. (4) and (5). Based on the results of our analysis of the energies $\Delta E_{\mathrm{C}}$ for more than 400 nuclei [8], one can state that the observed functional dependence corresponds to the SU(4)theory.

The most complete experimental studies of the entire spectrum of the charge-exchange excitations were performed in [6] for nine tin isotopes $112,114,116,117,118,119,120,122,124 \mathrm{Sn}$ exploiting the chargeexchange reaction $\left({ }^{3} \mathrm{He}, t\right)$ at beam energy $200 \mathrm{MeV}$. In particular, three charge-exchange pygmy resonances were identified in every $\mathrm{Sn}$ isotopes. Our results for the energies of five charge-exchange resonances AR, GTR, PR1, PR2, and PR3 for nine tin isotopes calculated within microscopic TFFS presented in Table 1 together with the experimental data from [6]. As it can be seen from Table 1, the standard r.m.s. deviations of the calculated from experimental ones for the energies are small enough $\delta E<0.40 \mathrm{MeV}$. These values of $\delta E$ are comparable with the experimental errors $\Delta E_{\text {exp }}= \pm 0.25 \mathrm{MeV}$. Our results are close to that of other calculations of high-lying excitations, e.g., performed in the framework of self-consistent QRPA with Skyrme forces [12].

Figure 2 demonstrates the difference between the energies of GTR and lying below pygmy resonances PR for Sn isotopes as functions of the mass number $A$. The calculations by Eq. (3) produces standard deviations $\delta E_{\mathrm{PR} 1}=0.53$ 
Table 1. Energies (in $\mathrm{MeV}$ ) of the analog $E_{\mathrm{AR}}$, Gamow-Teller $E_{\mathrm{GTR}}$, and three pygmy $E_{\mathrm{PR}}$ resonances calculated within the TFFS, corresponding experimental data [6], and the standard deviations $\delta E=<E_{\exp }-E_{\text {calc }}>$ of theoretical results from experimental data.

\begin{tabular}{|c|c|c|c|c|c|c|c|c|c|c|}
\hline Nucleus init./final & \multicolumn{2}{|c|}{$E_{\mathrm{AR}}$} & \multicolumn{2}{|c|}{$E_{\mathrm{GTR}}$} & \multicolumn{2}{|c|}{$E_{\mathrm{PR} 1}$} & \multicolumn{2}{|c|}{$E_{\mathrm{PR} 2}$} & \multicolumn{2}{|c|}{$E_{\mathrm{PR} 3}$} \\
\hline \multirow[b]{2}{*}{${ }^{112} \mathrm{Sn} /{ }^{112} \mathrm{Sb}$} & $\begin{array}{c}\text { exp. } \\
\pm 0.03\end{array}$ & calc. & $\begin{array}{l}\text { exp. } \\
\pm 0.25\end{array}$ & & $\begin{array}{c}\text { exp. } \\
\pm 0.25\end{array}$ & calc. & $\begin{array}{c}\text { exp. } \\
\pm 0.20\end{array}$ & calc. & $\begin{array}{c}\text { exp. } \\
\pm 0.20\end{array}$ & calc. \\
\hline & 6.16 & 6.69 & 8.94 & 9.38 & 4.08 & 4.70 & 2.49 & 3.00 & 1.33 & 1.52 \\
\hline${ }^{114} \mathrm{Sn} /{ }^{114} \mathrm{Sb}$ & 7.28 & 6.92 & 9.39 & 9.60 & 4.55 & 4.97 & 2.95 & 2.65 & 1.88 & 1.60 \\
\hline${ }^{116} \mathrm{Sn} /{ }^{116} \mathrm{Sb}$ & 8.36 & 8.47 & 10.04 & 10.36 & 5.04 & 5.23 & 3.18 & 2.68 & 1.84 & 1.75 \\
\hline${ }^{117} \mathrm{Sn} /{ }^{117} \mathrm{Sb}$ & 11.27 & 11.38 & 12.87 & 12.91 & 7.64 & 7.54 & 5.45 & 5.21 & 3.87 & 3.71 \\
\hline${ }^{118} \mathrm{Sn} /{ }^{118} \mathrm{Sb}$ & 9.33 & 9.23 & 10.61 & 10.93 & 5.38 & 5.54 & 3.17 & 3.08 & 1.47 & 1.55 \\
\hline${ }^{119} \mathrm{Sn} /{ }^{1{ }^{19}} \mathrm{Sb}$ & 12.36 & 12.48 & 13.71 & 13.77 & 8.09 & 8.27 & 5.49 & 5.57 & 3.63 & 4.07 \\
\hline${ }^{120} \mathrm{Sn} /{ }^{120} \mathrm{Sb}$ & 10.24 & 10.20 & 11.45 & 11.78 & 5.82 & 6.24 & 3.18 & 3.47 & 1.38 & 0.98 \\
\hline${ }^{122} \mathrm{Sn} /{ }^{122} \mathrm{Sb}$ & 11.24 & 11.17 & 12.25 & 12.54 & 6.65 & 6.76 & 3.37 & 3.91 & 1.45 & 1.55 \\
\hline${ }^{124} \mathrm{Sn} /{ }^{124} \mathrm{Sb}$ & 12.19 & 12.05 & 13.25 & 13.59 & 7.13 & 7.16 & 3.44 & 3.06 & 1.50 & 2.17 \\
\hline$\delta E$ & \multicolumn{2}{|c|}{0.23} & \multicolumn{2}{|c|}{0.29} & \multicolumn{2}{|c|}{0.31} & \multicolumn{2}{|c|}{0.36} & \multicolumn{2}{|c|}{0.33} \\
\hline
\end{tabular}

$\mathrm{MeV}, \delta E_{\mathrm{PR} 2}=0.26 \mathrm{MeV}$ and $\delta E_{\mathrm{PR} 3}=0.50 \mathrm{MeV}$ that is comparable with the microscopic calculations (see Table 1). Thus, on average the calculations by two methods describe the experimental data satisfactorily.

Charge-exchange strength functions $S\left(E_{x}\right)$ were calculated within TFFS for more than 100 isotopes in the middle-mass region. At present only few of these nuclides are investigated experimentally in charge-exchange nuclear reactions such as $(p, n)$ or $\left({ }^{3} \mathrm{He}, t\right)$. We present in Figure 3a) the experimental data on excitation spectra obtained in ${ }^{118} \mathrm{Sn}\left({ }^{3} \mathrm{He}, t\right){ }^{118} \mathrm{Sb}$ reaction [6]. The calculated charge-exchange strength function $S\left(E_{x}\right)$ for ${ }^{118} \mathrm{Sn}$ is depicted in Figure 3b). Unfortunately, direct measurements of the strength function $S\left(E_{x}\right)$ were not performed, but the data on counts shown in Figure 3a) are proportional to the partial data on the function $S\left(E_{x}\right)$. One more example of the experimental and theoretical strength functions are given in Figure 4 for the ${ }^{71} \mathrm{Ga}$ nucleus. Here, the GTR resonance as well as three pygmy resonances located at lower excitation energy are seen as well. Theoretical and experimental energies of the resonances are in reasonable agreement. These resonances play significant role in the neutrino capturing reactions as will be discussed in the next section 4.

\section{Charge-exchange resonances in the neutrino capturing reactions}

The charge-exchange resonances strongly influence neutrino capturing cross sections by nuclei $\sigma\left(E_{v}\right)$. We calculated within TFFS charge-exchange strength functions $S\left(E_{x}\right)$ for ${ }^{71} \mathrm{Ga},{ }^{98} \mathrm{Mo}$ and ${ }^{127} \mathrm{I}$ nuclei. Then, knowing $S\left(E_{x}\right)$ we calculated cross sections $\sigma\left(E_{v}\right)$ applying the following equation (see [14]):

$$
\begin{aligned}
& \sigma\left(E_{v}\right)=\frac{\left(G_{\mathrm{F}} g_{A}\right)^{2}}{\pi c^{3} \hbar^{4}} \int_{0}^{E_{v}-Q} E_{e} p_{e} F\left(Z, A, E_{e}\right) S(x) d x \\
& E_{e}=E_{v}-Q-x+m_{e} c^{2}, \quad c p_{e}=\sqrt{E_{e}^{2}-\left(m c^{2}\right)^{2}} .
\end{aligned}
$$

In Eq.(7), $E_{v}$ is an incident neutrino energy, $F\left(Z, A, E_{e}\right)-$ the Fermi function, $G_{\mathrm{F}} /(\hbar c)^{3}$ - the Fermi weak interaction constant, $g_{A}-$ the axial-vector constant from [15]. Cal-

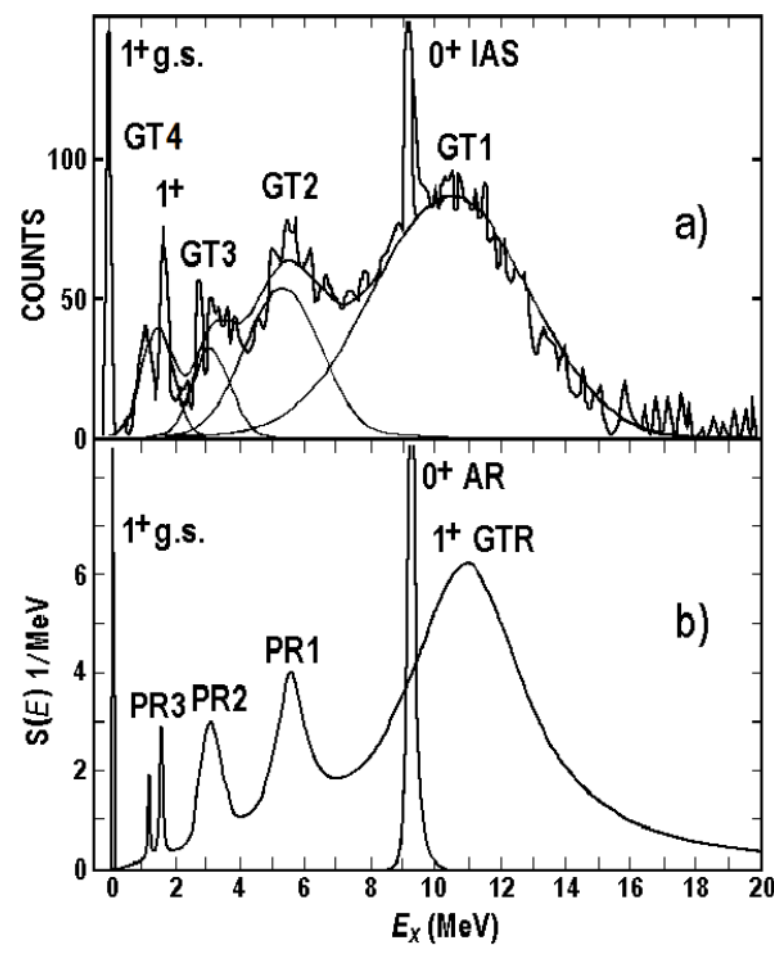

Figure 3. Panel a) - experimental spectrum of the reaction ${ }^{118} \mathrm{Sn}\left({ }^{3} \mathrm{He}, t\right){ }^{118} \mathrm{Sb}[6]$. Panel b) - calculated charge-exchange strength function $S(E)$. The Gamow-Teller and three pygmy resonances are marked as GT1, GT2, GT3 and GT4 in panel a) and GTR, PR1, PR2, and PR3 in panel b).

culations were carried out for ${ }^{71} \mathrm{Ga},{ }^{98} \mathrm{Mo}$ and ${ }^{127} \mathrm{I}$ nuclei which are of interest for neutrino physics. The results of calculations $\sigma\left(E_{v}\right)$ are shown partly in Figure 5. It is seen that the effect of charge-exchange resonances on the cross section is quite sizable. Even at $E_{v}=4 \mathrm{MeV}$, the exclusion of the Gamow-Teller resonance alone leads to reduction in the cross section by $25 \%$ for the reaction ${ }^{71} \mathrm{Ga}\left(v_{e}, e^{-}\right)^{71} \mathrm{Ge}$. For the ${ }^{98} \mathrm{Mo}\left(v_{e}, e^{-}\right)^{98} \mathrm{Tc}$ reaction exclusion of GTR reduces the cross-section by $16 \%$ at $E_{v}=6 \mathrm{MeV}$ and by $45 \%$ at $E_{v}=14 \mathrm{MeV}$. For the ${ }^{127} \mathrm{I}\left(v_{e}, e^{-}\right)^{127} \mathrm{Xe}$ reaction the crosssection appears to be reduced by $19 \%$ at $E_{v}=2 \mathrm{MeV}$ and by $69 \%$ at $E_{\nu}=14 \mathrm{MeV}$. Certainly, the effect becomes stronger 


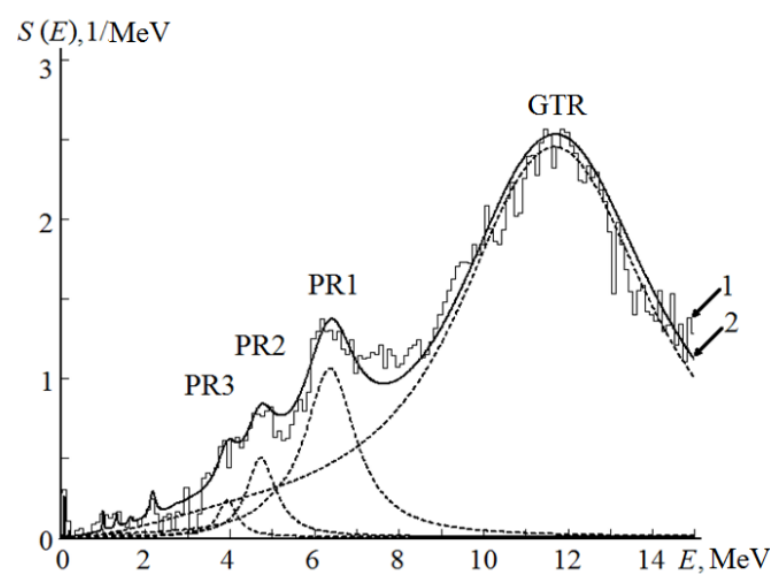

Figure 4. Charge-exchange strength functions $S(E)$ for the ${ }^{71} \mathrm{Ga}$ nucleus. 1 (bar chart) - experimental data [13], 2 (solid line) numerical calculations within TFFS. GTR - the Gamow-Teller resonance, PR1, PR2, PR3 - pygmy resonances.

if one disregards not only GTR, but PR1 in addition. The elimination of two resonances, GTR and PR1, reduces the cross section of the ${ }^{71} \mathrm{Ga}\left(v_{e}, e^{-}\right){ }^{71} \mathrm{Ge}$ reaction by an amount varying from $20 \%$ to $60 \%$ with a change in the neutrino energy from 2 to $14 \mathrm{MeV}$. From Figure 5 one can see that the strongest effect of GTR and PR resonances on $\sigma(E)$ is observed for the reaction ${ }^{127} \mathrm{I}\left(v_{e}, e^{-}\right){ }^{127} \mathrm{Xe}$.

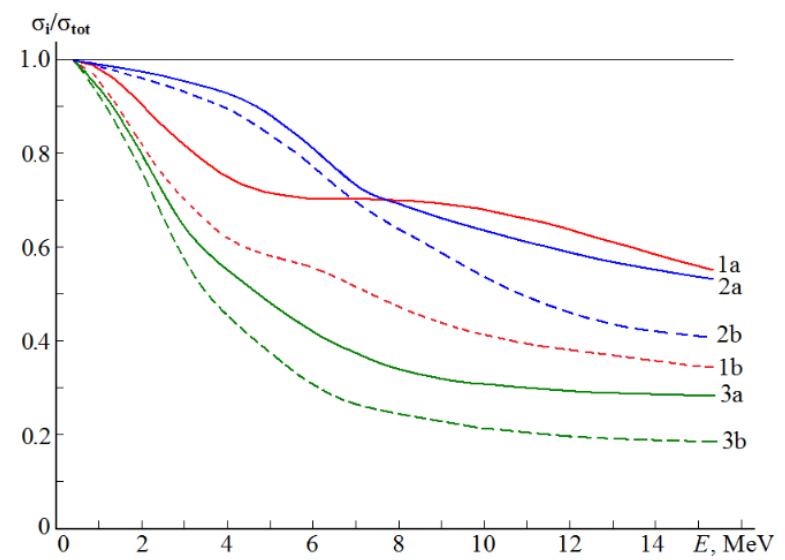

Figure 5. (Color online) The effect of GTR and PR1 resonances on the cross section $\sigma_{i}(E)$ of neutrino capturing on ${ }^{71} \mathrm{Ga},{ }^{98} \mathrm{Mo}$ and ${ }^{127} \mathrm{I}$ nuclei. The ratio $\sigma_{i}(E) / \sigma_{\text {tot }}$ calculated within TFFS is shown as a function of neutrino energy $E_{v} . \sigma_{\text {tot }}-$ cross section calculated with the complete strength function $S\left(E_{v}\right)$. Solid lines - calculations of $\sigma_{i}(E)$ with exclusion the GTR contribution only. Dashed lines - calculations of $\sigma_{i}(E)$ with exclusion both GTR and PR1. 1 (red) - calculations for ${ }^{71} \mathrm{Ga}\left(v_{e}, e^{-}\right)^{71} \mathrm{Ge}$ reaction, 2 (blue) - calculations for ${ }^{98} \mathrm{Mo}\left(v_{e}, e^{-}\right)^{98} \mathrm{Tc}$ reaction, 3 (green) -calculations for ${ }^{127} \mathrm{I}\left(v_{e}, e^{-}\right)^{127} \mathrm{Xe}$ reaction.

\section{Conclusions}

Observed in all charge-exchange reactions three types of the isobaric resonances - the giant Gamow-Teller (GTR), the analog (AR) and pygmy (PR) ones - are investigated exploiting the microscopic theory of finite Fermi systems and its approximate version. The calculated energies of GTR, AR and three PRs are in good agreement with the experimental data. The root-mean-square deviation for the value $\Delta E_{\mathrm{G}-\mathrm{A}}$ is $0.30 \mathrm{MeV}$ for the 33 considered nuclei where experimental data are available. The comparison of calculations with experimental data on the energies of charge-exchange pygmy resonances gives the standard deviation $\delta E<0.40 \mathrm{MeV}$. Calculated difference of energies of GTR and AR resonances $\Delta E_{\mathrm{G}-\mathrm{A}}=E_{\mathrm{GTR}}-E_{\mathrm{AR}}$ go to zero in heavier nuclei indicating the restoration of Wigner SU(4)symmetry. This allows to describe the properties of heavy nuclei more confidently using SU(4)-theory, especially for mass relations [16]. Also the analysis of the Coulomb displacement energies using the SU(4)-approach allows to describe AR energies and the masses of superheavy nuclei with a good accuracy.

Strength functions for the ${ }^{118} \mathrm{Sn},{ }^{71} \mathrm{Ga},{ }^{98} \mathrm{Mo}$ and ${ }^{127} \mathrm{I}$ nuclides were calculated and analyzed. The calculated resonance energies and amplitudes of resonance peaks appear to be close to the experimental values. Strong influence of the charge-exchange resonances on neutrino capturing cross sections is demonstrated.

Further investigation of the resonant structure of the strength functions $S(E)$ will allow us to analyze the data of the charge-exchange reactions and $\beta$-delayed processes. So the appearance of PR in the energy window of the emission of $\beta$-delayed neutrons leads to a sharp increase in the probability of this process [17]. Similar effect can be observed for the $\beta$-delayed fission process [18].

The author is grateful to I.N. Borzov, V.N. Tikhonov, E.E. Saperstein and S.V. Tolokonnikov for their assistance and helpful discussions. The work is supported by the Russian Foundation for Basic Research (grants 16-02-00228, 18-02-00670).

\section{References}

[1] Yu.V. Gaponov, Yu.S. Lutostanskii, JETP Lett. 15, 120 (1972)

[2] Yu.V. Gaponov, Yu.S. Lutostanskii, Sov. J. Nucl. Phys. 19, 33 (1974)

[3] R.R. Doering, A. Galonsky, D.M. Patterson, G.F. Bertsch, Phys. Rev. Lett. 35, 1691 (1975)

[4] A. Galonsky, R.R. Doering, D.M. Patterson, H.W. Bertini, Phys. Rev. C 14, 748 (1976)

[5] Yu.S. Lutostansky, JETP Lett. 106, 7 (2017)

[6] K. Pham, J. Jänecke, D.A. Roberts, M.N. Harakeh et al., Phys. Rev. C 51, 526 (1995)

[7] A.B. Migdal, Theory of Finite Fermi Systems and Applications to Atomic Nuclei (Interscience Publishers, New York, 1967)

[8] Yu.S. Lutostansky, V.N. Tikhonov, JETP Lett. 102, 7 (2015); Phys. At. Nucl. 79, 929 (2016)

[9] Yu.S. Lutostansky, V.N. Tikhonov, Bull. Russ. Acad. Sci. Phys. 79, 425 (2015); Phys. At. Nucl. 80, 623 (2017)

[10] P. Möller, J.R. Nix, W.D. Myers, W.J. Swiatecki, At. Data \& Nucl. Data Tables 59, 185 (1995)

[11] Yu.S. Lutostansky, V.N. Tikhonov, Bull. Russ. Acad. Sci. Phys. 78, 373 (2014)

[12] I.N. Borzov, S. Goriely, Phys. Rev. C 62, 035501 (2000) 
[13] D. Krofcheck, E. Sugarbaker, J. Rapaport, D. Wang, J.N. Bahcall et al., Phys. Rev. Lett. 55, 1051 (1985)

[14] Yu.S. Lutostansky, N.B. Shul'gina, Phys. Rev. Lett. 67, 430 (1991)

[15] C. Patrignani, Particle Data Group, Chin. Phys. C 40, 100001 (2016)
[16] Yu.V. Gaponov, N.B. Shul'gina, D.M. Vladimirov, Nucl. Phys. A 391, 93 (1982)

[17] D. Verney, D. Testov, F. Ibrahim, Yu. Penionzhkevich et al., Phys. Rev. C 95, 054320 (2017)

[18] Yu.S. Lutostansky, V.I. Lyashuk, JETP Lett. 107, 79 (2018) 